\title{
The effect of a mouthrinse containing essential oils on dental plaque and gingivitis
}

\author{
Do mouthrinses containing essential oils reduce the effect of dental plaque \\ and gingivitis in the long term?
}

\author{
Stoeken J, Paraskevas S, van der Weijden G. \\ The long-term effect of a mouthrinse containing essential oils on \\ dental plaque and gingivitis: A systematic review. J Periodontol. \\ 2007;78: 1218-1228
}

Data sources: PubMed and the Cochrane Central Register of Controlled Trials were searched up to December 2006 were searched. Only studies published in English were included.

Study Selection: Randomised controlled clinical trials, controlled clinical trials and uncontrolled longitudinal clinical trials were included in the initial search. Studies with a minimum duration of 6 months, healthy subjects $\geq 18$ and gingivitis without severe periodontal disease were included. The effects of plaque and gingivitis were considered the primary outcomes with staining of teeth a secondary outcome.

Data extraction and synthesis: Studies were screened and data extracted independently by two reviewers. It is unclear whether or not this process was duplicated. Disagreements were resolved by discussion. Heterogeneity of the studies was assessed. Data was pooled for gingivitis and plaque and a weighted means meta-analysis using a random effects model was carried out.

Results: Eleven studies (all randomised controlled trials) met the inclusion criteria. All were of six months duration except one of nine months. There was no meta-analysis between baseline and end trial as the standard deviation could not be calculated. Three studies were not included in the meta-analysis. Meta-analysis of staining was not carried out. There was significant reduction in gingivitis with EO mouthrinses compared to control groups regardless of the measurement index used (Weighted Means Difference (WMD) - 0.32 95\% Confidence Interval (Cl) [-0.46 to $-0.19], P<0.00001$; test for heterogeneity: $\left.\mathrm{P}<0.00001 \mathrm{I}^{2}=96.7 \%\right) . \mathrm{A}$ significant reduction in interproximal gingivitis was also noted for EO mouthrinses compared to control (WMD $-0.2995 \% \mathrm{Cl}$ [-0.48 to -0.11$]$ $\mathrm{P}=0.002$; test for heterogeneity: $\left.\mathrm{p}<0.0001 \mathrm{I}^{2}=95.8 \%\right)$ and compared to floss (WMD $-0.0595 \% \mathrm{Cl}[-0.20$ to -0.09$] \mathrm{P}=0.48$; test for heterogeneity: $\left.\mathrm{P}=0.0001 \mathrm{I}^{2}=99.7 \%\right)$. Similar results were seen for the effects on plaque with a decrease in total plaque in favour of EO mouthrinse (WMD - 0.83 95\% Cl [-1.13 to -.053$] \mathrm{P}<0.00001$; test for heterogeneity: $\mathrm{P}<0.00001$ $\left.I^{2}=96.1 \%\right)$. Significant interproximal plaque reduction, again in favour of EO mouthrinse, was also seen compared to control (WMD -1.02 [1.44 to -0.60$] \mathrm{P}<0.00001$; test for heterogeneity: $\mathrm{P}<0.00001 \mathrm{I}^{2}=96.1 \%$ $95 \% \mathrm{Cl}$ ) and compared to floss (WMD $-0.7595 \% \mathrm{Cl}[-1.15$ to -0.363$]$ $\mathrm{P}<0.0002$; test for heterogeneity: $\mathrm{P}<0.0002 \mathrm{I}^{2}=93 \%$ )

Conclusion When used as an adjunct to unsupervised oral hygiene, the existing evidence supports that essential oil provides an additional benefit with regard to plaque and gingivitis reduction compared to placebo or control.

Address for correspondence: Dr. G.A. van der Weijden, Department of Periodontology, Academic Center for Dentistry Amsterdam, Louwesweg 1, 1066 EA Amsterdam, The Netherlands.; e-mail: ga.vd.weijden@acta.nl

\section{Commentary}

This review aimed to investigate the long term effect of a mouthrinse Containing essential oils (Listerine, Pfizer Consumer Healthcare, Morris Plains, New Jersey, USA) on gingivitis and dental plaque. Overall, this review has been carried out in a structured manner. There are however some important considerations to bear in mind when using the results from the analysis.

Although two major electronic databases were searched, many other sources were neglected. The search also restricted studies to those published in English. Some indication of the number of studies excluded on the basis of language would have been interesting. Unpublished studies were also not included in this review. All of these factors may combine to introduce an element of publication bias; studies in which an intervention is effective are more likely to be published. Therefore searching only (part of) the published literature may overestimate the effect of an intervention. The authors make no mention of addressing the possibility of publication bias either through the use of funnel plots or other methods. There were only two reviewers to screen and review the studies, hence increasing the risk of error. Further to this, eight of the eleven studies included in the review were either funded by or associated with the company that manufactures a well known and widely available essential oil-containing mouthrinse.

There was no mention of the reasons for drop outs in any of the studies. Some other data were also missing which prevented certain statistical analysis. There did not appear to be any attempt made to contact the authors of the studies included regarding the missing data.

The relevance and accuracy of results from meta-analyses are dependent on the figures entered. The indices used to measure plaque and gingivitis scores were different between studies (six of which used a Modified Gingival Index and 5 using the Gingival Index to measure gingivitis) and poses the question is a meta-analysis appropriate in this case? There were a number of other differences between the studies reviewed. A variety of controls were used: water, hydroalcohol control, vehicle control, $0.12 \%$ chlorhexidine. Two studies included flossing as a control whilst one study used floss in conjunction with an essential-oil mouthrinse as the intervention. The heterogeneity between studies was noted as being significant at the end point, and as the authors of the review note, caution must be exercised when using WMD values as an exact measure of effect.

Interestingly, the only study ${ }^{1}$ in the review that showed there was no significant effect of EO mouthrinses on gingivitis was not able to be included in the meta-analysis due to a lack of baseline and end point standard deviation figures after standard deviation. Another study ${ }^{2}$ included in the analysis showed a significant difference at the end point of nine months but closer examination of this study reveals that at the six month period there was no significant reduction in plaque or gingivitis demonstrated by essential-oil mouthrinse. But it is after this, when there is a large drop out of subjects from the 
study, and the remaining subjects were no longer supervised when rinsing with the essential-oil mouthrinse, that a significant reduction is seen. It is less certain what the effects may be in a less controlled setting such as the general population.

Consideration should also be given to the possibility of the Hawthorne effect influencing findings in these studies. Participants of the studies may have improved their oral hygiene standards by merely taking part in the study regardless of intervention. Again, the authors of the review identify this point. An issue that appears to have been overlooked is the concentration of the oils . It is far from clear what concentrations were used in the studies selected. Certainly, differing concentrations of $\mathrm{EO}$ would bring into question the validity of the results of this meta-analysis.

The authors do provide a reasonable discussion of their own, regarding some other drawbacks and important considerations of their study. Whilst their conclusion is appropriate for the results of their review and analysis, a different set of results and possibly a different conclusion may have been drawn if a wider search had been carried out and further attention paid to the issues raised above.

\section{Rajiv M Patel and Zainab Malaki}

Periodontology Department of Periodontology, Kings College

London, Dental Institute at Guy's, Hospital, London SE1 9RT

1. Grossman E, Meckel AH, Isaacs RL, et al. A clinical comparison of antibacterial mouthrinses: Effects of chlorhexidine, phenolics, and sanguinarine on dental plaque and gingivitis. J Periodontol 1989;60:435-440.

2. Gordon JM, Lamster IB, Seiger MC. Efficacy of Listerine antiseptic in inhibiting the development of plaque and gingivitis. J Clin Periodontol 1985;12: 697-704.

Evidence-Based Dentistry (2008) 9, 18-19. doi:10.1038/sj.ebd.6400566

\section{Key to evidence graphic used in the Evidence-based Dentistry Journal}

The graphic is based on the Centre for Evidence-based Medicine levels of Evidence tables www.cebm.net/levels_of_evidence.asp (see Evidence-based Dentistry 2003;4: p 17-18)

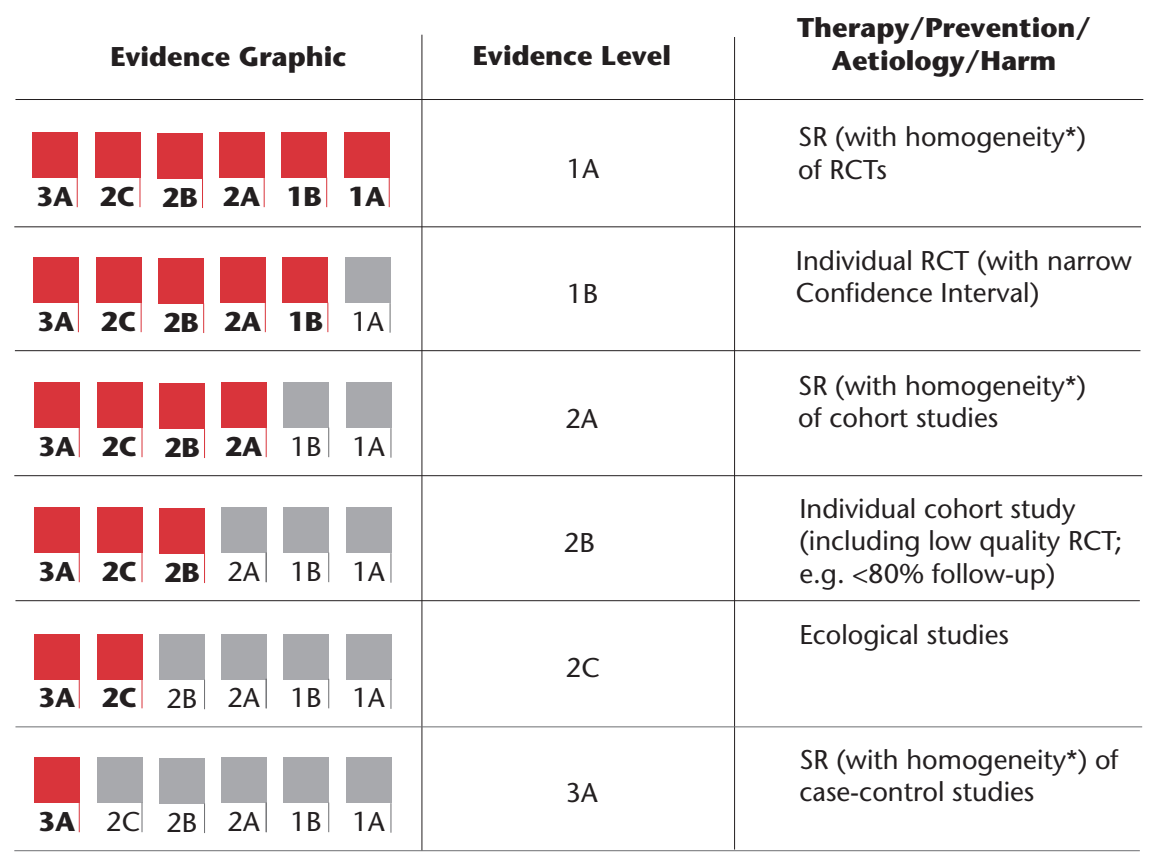

* By homogeneity we mean a systematic review that is free of worrisome variations (heterogeneity) in the directions and degrees of results between individual studies. Not all systematic reviews with statistically significant heterogeneity need be worrisome, and not all worrisome heterogeneity need be statistically significant. 